\title{
PABA Release from Chitosan-PCL with Induced Electric Current
}

\author{
Jennifer M Miller and Roche C de Guzman* \\ Department of Engineering, Hofstra University, USA
}

Received: 制: July 24, 2018; Published: 畊 July 31, 2018

*Corresponding author: Roche C de Guzman,133 Hofstra University, 203 Weed Hall, Hempstead, NY 11549, USA

\begin{abstract}
Controlled drug delivery systems such as the stimulation-based biomaterial scaffolds for sequestration and release of drugs offer safety and regulated therapeutic approach. In this study, the drug: para-aminobenzoic acid (PABA) was absorbed into a crosslinked chitosan and poly(caprolactone) (PCL) hydrogel and its release kinetics quantified under different conditions. It was experimentally-observed that the higher the $\mathrm{pH}$ (or the more basic the $\mathrm{pH}$ ), the slower the PABA saturation release trended over time. At the acidic environment of pH 4, PABA was released the fastest, and enhanced by the degradation of chitosan-PCL gel. When a constant electric current of $0.6 \mathrm{~mA}$ as applied, PABA release was induced at $\mathrm{pH}$ 10. However, at pH 7, PABA was stably-bound to the chitosan-PCL matrix, with or without the external current. The selective sequestration of PABA at basic $\mathrm{pH}$ and its stimulated release via electric current application can be further explored for clinical translatability.
\end{abstract}

Keywords: Drug Delivery; Chitosan; PCL; Biomaterial Scaffolds; PABA; Electrical Stimulation; Electrostatic Interaction

Abbreviations: DDS: Drug Delivery Systems; PCL: Poly(Caprolactone); PABA: Para-Aminobenzoic Acid; DCC: N,N’-Dicyclohexylcarbodiimide; DBTDL: Dibutyltin Dilaurate; DMACA: 4-(Diethylamino)Cinnamaldehyde; MOPS: 4-Morpholinepropanesulfonic Acid

\section{Introduction}

Controlled drug delivery systems (DDS) are smart devices regulating the sequestration, release, and bioavailability of therapeutics. Clinically-successful DDS materials include chitosan and poly(caprolactone) (PCL) as they impart safety and biocompatibility $[1,2]$. Importantly, chitosan provides protonizable amine groups, enabling matrix electrostatic interaction of anionic and negatively-charged molecules [3]. Crosslinking of PCL with chitosan enables tunability of mechanical and degradation properties without affecting the DDS net charge since PCL is neutral [4-6]. Release of chitosan-bound drugs can be induced via stimulation of external electric field [7-9]. In this study, the absorption and release kinetics of para-aminobenzoic acid (PABA) to and from crosslinked chitosan-PCL scaffold, respectively, was characterized under different $\mathrm{pH}$ conditions. PABA, a benzene ring with para (carbon 1 and 4) positions of amine and carboxylic acid groups, is a zwitterion that changes in charge depending on the buffer $\mathrm{pH}$. Due to its multiple pKa values [10] as well as its complex interaction with the charged chitosan-PCL matrix, PABA's dynamic behavior is challenging to model, hence experimental outcome is needed. Moreover, PABA release was quantified in the presence of sub-milliampere electric current for the implementation of a controlled DDS with medicinal implications $[11,12]$.

\section{Materials and Methods}

\section{Reagents}

Para-aminobenzoic acid (PABA), poly(caprolactone) (PCL; Mn 80000), N,N'-dicyclohexylcarbodiimide (DCC), dibutyltin dilaurate (DBTDL), 4-(dimethylamino)cinnamaldehyde (DMACA), sodium acetate, 4-morpholinepropanesulfonic acid (MOPS), sodium tetraborate decahydrate (sodium borate), acetic acid, and $\mathrm{NaOH}$ were purchased from Sigma-Aldrich (St. Louis, MO). Chitosan (70\% deacetylated) was obtained from Primex (Siglufjordur, Iceland). Deionized water $(18.2 \mathrm{M} \Omega \cdot \mathrm{cm}$ resistivity) was used as the solvent, unless stated otherwise. Three buffers at different $\mathrm{pH}$ were prepared: $81 \mathrm{mM}$ sodium acetate ( $\mathrm{pH} 4), 100 \mathrm{mM}$ MOPS (pH 7), and $50 \mathrm{mM}$ sodium borate ( $\mathrm{pH} 10)$.

\section{Chitosan-PCL Hydrogel Fabrication}

Solutes: chitosan $(40 \mathrm{mg} / \mathrm{mL})$ and PCL $(10 \mathrm{mg} / \mathrm{mL})$ and crosslinkers: DCC $(1 \mathrm{mg} / \mathrm{mL})$ and DBTDL $(0.07 \mu \mathrm{L} / \mathrm{mL})$ were dissolved and reacted in $80 \%$ acetic acid at $37{ }^{\circ} \mathrm{C}$ (modified from Aroguz et al. [4]) in 24-well microplates (1mL / well). Water was evaporated inside the fume hood. Scaffolds were soaked and rinsed in $1 \mathrm{M} \mathrm{NaOH}$ for two days to stabilize, then in multiple water washes until $\mathrm{pH}$ 7. Crosslinked chitosan-PCL were equilibrated in

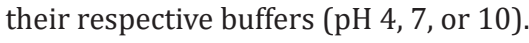




\section{PABA Absorption and Release}

$1 \mathrm{mg} / \mathrm{mL}$ PABA in $\mathrm{pH}$ buffers were added to their corresponding equilibrated chitosan-PCL for 2 hours to allow for PABA absorption into the bulk. The unabsorbed liquid was collected for indirect measurement of the gel-absorbed PABA (absorbed = mass added - mass unabsorbed). Gels were quickly rinsed in buffers to remove any residual unabsorbed PABA. $1 \mathrm{~mL}$ buffer was added at $0.5,1,2$, and 4 hours, then the fluid was aspirated out and collected, and the scaffold was replenished with fresh $1 \mathrm{~mL}$ buffer. Release groups containing no absorbed PABA were used as controls.

Combined chitosan and PABA levels from the collected samples were quantified via a colorimetric reaction with DMACA (amine detection) [13] and absorbance read at 490nm (for pH 4 and 7) and at $595 \mathrm{~nm}$ (for $\mathrm{pH} 10$ ) using a spectrophotometer (iMark Reader, Bio-Rad, Hercules, CA). Chitosan release was obtained using the no-PABA (negative) controls. PABA quantities were calculated by subtracting the chitosan from the combined chitosan and PABA values.

\section{Electric Current Application}

The electrical system was assembled based on Sheen and de Guzman using a MATLAB (MathWorks, Natick, MA)-controlled Arduino [14]. Briefly, electrodes spaced $1.5 \mathrm{~cm}$ apart (across the gel) were inserted into the scaffolds with absorbed PABA in buffers pH 7 and 10. A continuous current of $0.6 \mathrm{~mA}$ was applied, and liquids were collected at $0,2,6,10,14$, and 18-minute intervals. Fresh buffer was added per time point. Controls were tested at no applied electric current.

\section{Statistical Analyses}

Experimental samples were performed in triplicates $(n=3)$. Values were reported as average \pm 1 standard deviation. Saturation regression equations were fitted using least-squares method in MATLAB:

$$
C(t)=C_{\max }\left(\frac{t}{t_{h}+t}\right)
$$

where, $\mathrm{t}=$ time $[\mathrm{hr}], \mathrm{C}=$ cumulative mass release concentration, $\mathrm{C}_{\max }=$ theoretical maximum concentration, and $\mathrm{t}_{\mathrm{h}}=$ time to reach half $\mathrm{C}_{\max }$. Scatterplots and one-way analysis of variance (ANOVA) with multiple comparison (Tukey's) were made in MATLAB at 5\% probability (p) of type I error.

\section{Results}

\section{Chitosan-PCL Gels}

The fabricated chitosan-PCL scaffolds were stable in water at $\mathrm{pH} 7$ and initially after equilibration with the different buffers (Figure 1a). However, after hours of incubation, those in acidic medium ( $\mathrm{pH} 4$ ) appeared to degrade faster relative to gels in higher $\mathrm{pH}$ values. DMACA colorimetric assay confirmed that the aminecontaining chitosan chains were released most in the acidic buffer.

\section{PABA Release Kinetics}

Chitosan-PCL gels absorbed $0.26,0.82$, and $1.73 \mathrm{mg}$ PABA, at

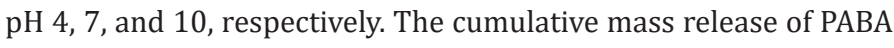
normalized to these initial masses produced saturation curves (Figure 1b) with the fitted least-squares regression equation, $C(t)=C_{\max }\left(\frac{t}{t_{h}+t}\right)$ constants: $\mathrm{C}_{\text {max }}=1.0328$ and th $=0.1320 \mathrm{hr}\left(\mathrm{r}^{2}=\right.$ $0.93)$ at $\mathrm{pH} 4, \mathrm{C}_{\max }=0.7060$ and $\mathrm{t}_{\mathrm{h}}=0.548 \mathrm{hr}\left(\mathrm{r}^{2}=0.83\right)$ at $\mathrm{pH} 7$, and $\mathrm{Cmax}=0.2387$ and th $=1.1565 \mathrm{hr}\left(\mathrm{r}^{2}=0.96\right)$ at $\mathrm{pH} 10$. The th (time to achieve half of Cmax) values showed increasing trend as $\mathrm{pH}$ increased. Based on the saturation functions at the 4-hour time point, the amount of PABA released into the liquid media were $100 \%$ at $\mathrm{pH} 4,62.1 \%$ at $\mathrm{pH}$ 7, and $18.5 \%$ at $\mathrm{pH} 10$ (p $\leq 0.0291$; significantly lower release compared to $\mathrm{pH} 4$ and 7).

(a)

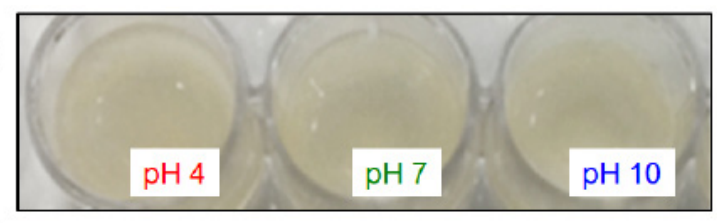

(b)

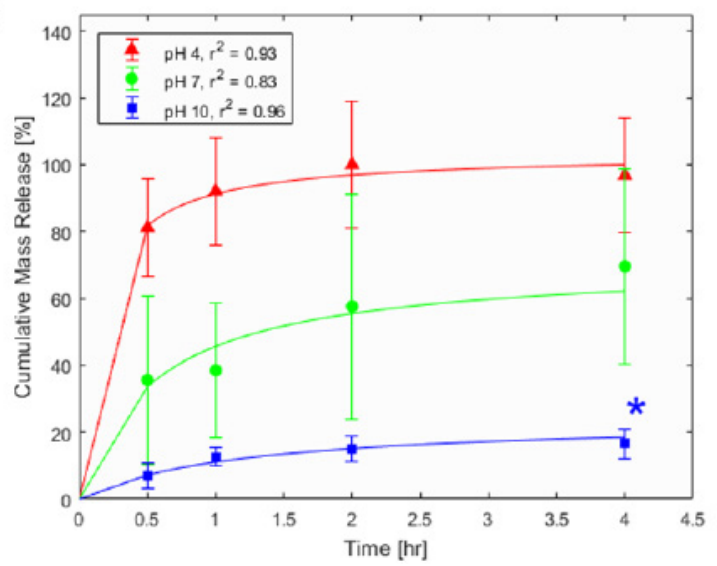

Figure 1: (a) Chitosan-PCL gels absorbed with PABA displayed (b) different saturation release kinetics of PABA in buffers with $\mathrm{pH}$ of 4,7 , and $10 .{ }^{*} \mathrm{p} \leq 0.0291$ compared to the other groups. 


\section{PABA Release with Electric Current Induction}

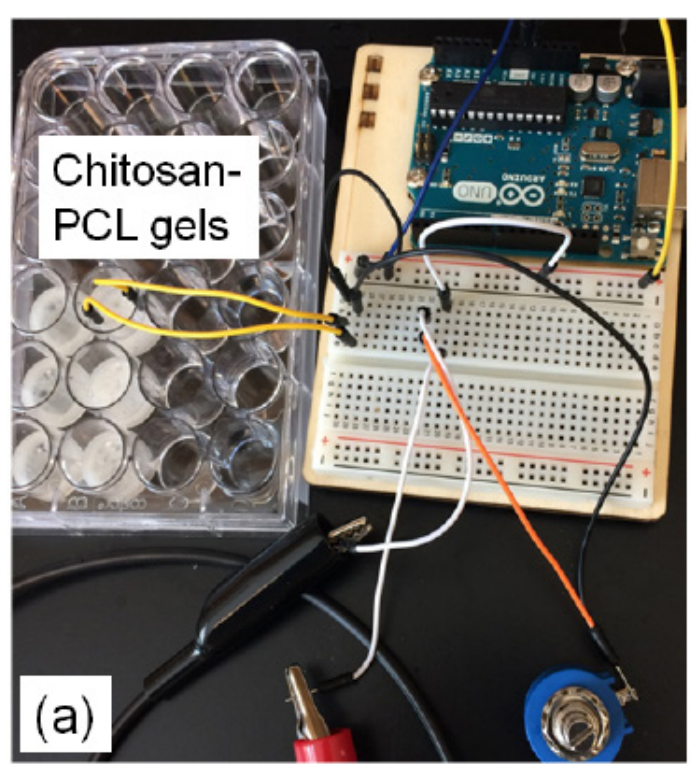

(b)

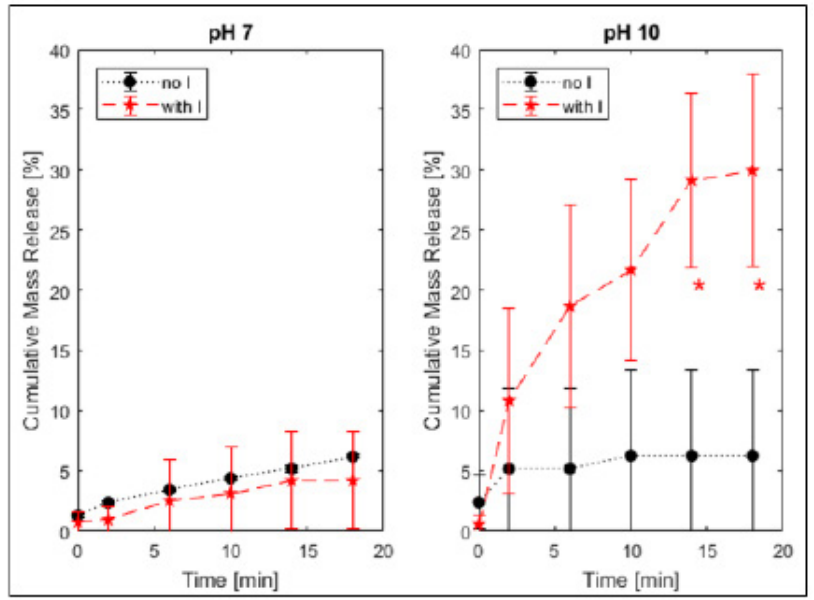

Figure 2: (a) Experimental setup for $0.6 \mathrm{~mA}$ electric current delivery (with I) into the chitosan-PCL gels to (b) release PABA. Control gels without current (no I) were compared side-by-side at $\mathrm{pH} 7$ and $10 .{ }^{*} \mathrm{p} \leq 0.0028$ compared to the no I group.

The chitosan-PCL gel system (Figure 2a), in different $\mathrm{pH}$ buffers, with electrode separation distance of $1.5 \mathrm{~cm}$ generated electrical resistance values in the $8 \mathrm{k} \Omega$ range. Consequently, the highest electrical current generated with the $5-\mathrm{V}$ potential of the Arduino controller was at $0.6 \mathrm{~mA}$. Application of this continuous current for 18 minutes led to different PABA release profiles (Figure $2 \mathrm{~b}$ ). At the neutral $\mathrm{pH}$ of 7 , the release kinetics of PABA, with and without electric current were statistically similar $(p \geq 0.6479)$ at all time points. The longer the time, the more mass was freed from the chitosan-PCL bulk into the medium; and by 14 and 18 minutes, the release was statistically-greater $(p \leq 0.0028)$ relative to the control group without electric current application.

\section{Discussion}

The crosslinking conditions (such as solvent type and concentration, crosslinker amount, and reaction times) using DCC and DBTDL with chitosan and PCL chains can be improved to fabricate a more stable gel network that can be utilized to deliver drugs. In our current system, the unbound chitosan affected the PABA absorption and release kinetics; hence, increased datapoint variability (Figure 1b \& Figure 2b). Experimental results showed (Figure $1 \mathrm{~b}$ ) that the higher the $\mathrm{pH}$, the more PABA associated with the chitosan network suggesting electrostatic interaction effects. At basic $\mathrm{pH}$, the positively-charged chitosan sequestered the highly negatively-charged PABA with acidic isoelectric point [15]. The PABA diffusion out of the chitosan-PCL network represented $78 \%$ to $100 \%$ total release relative to the Cmax (theoretical maximum asymptote) values indicating that, at the neutral and basic $\mathrm{pH}$ media, the network-bound PABA was stable and cannot be dissociated by diffusion-mediated forces.

The complete release of PABA in the acidic $\mathrm{pH}$ environment may be attributed to the repulsion between the highly positivelycharged chitosan network and the positively-charged protonated amine group of PABA. In the presence of an external current (Figure 2b), the PABA release at $\mathrm{pH} 7$ was found to be similar to the "no current" control. This observation suggests that PABA was neutral, thus negative charges from the electric current did not alter its mass release. However, at $\mathrm{pH} 10$, the release behavior of PABA was found to be time-dependent. A possible mechanism is that, at $\mathrm{pH}$ 10 , flowing electrons displaced the negatively-charged PABA from the positive chitosan-based matrix [8] leading to induced PABA release via electro osmosis and diffusion. This electro responsive approach [9] can be a useful controlled drug delivery strategy.

\section{Conclusion}

The amphoteric PABA is shown to have altered interactions with chitosan-PCL scaffolds based on $\mathrm{pH}$ values of the liquid media. More PABA is absorbed but less is released at higher pH. Electric current induces the release of tightly-bound PABA (at basic $\mathrm{pH}$ ), likely due to electrostatic competition of negatively-charged PABA and flowing electrons. This controlled response can be utilized for the development of targeted PABA therapeutics and nutritional usage $[11,12]$.

\section{Acknowledgement}

We would like to thank the members of the Bioengineering Materials Lab for their technical assistance, manuscript proofreading, and discussion, Jacqueline Scarola and Lori Castoria for help with purchasing, and Sina Rabbany for his continuing support and collaboration in conducting biomaterials research at Hofstra SEAS. Support for this study was provided by Hofstra University internal research funds.

\section{References}

1. Mohammed MA, Syeda JTM, Wasan KM and Wasan EK (2017) An Overview of Chitosan Nanoparticles and Its Application in NonParenteral Drug Delivery. Pharmaceutics 9(4): 53. 
2. Li Z, Tan BH (2014) Towards the development of polycaprolactone based amphiphilic block copolymers: molecular design, self-assembly and biomedical applications. Materials science \& engineering. C, Materials for biological applications 45: 620-634.

3. Kumar MN, Muzzarelli RA, Muzzarelli C, Sashiwa H, Domb AJ (2004) Chitosan chemistry and pharmaceutical perspectives. Chem Rev 104(12): 6017-6084.

4. Aroguz AZ, Baysal K, Tasdelen, Baysal BM (2011) Preparation, characterization, and swelling and drug release properties of a crosslinked chitosan-polycaprolactone gel. Journal of Applied Polymer Science 119(5): 2885-2894.

5. Ozkan O, Sasmazel HT (2018) Antibacterial Performance of PCLChitosan Core-Shell Scaffolds. J Nanosci Nanotechnol 18(4): 2415-2421.

6. Woodruff MA, Hutmacher DW (2010) The return of a forgotten polymerPolycaprolactone in the 21st century. Prog Polym Sci 35(10): 1217-1256.

7. Alvarez Lorenzo C, Blanco Fernandez B, Puga AM, Concheiro A (2013) Crosslinked ionic polysaccharides for stimuli-sensitive drug delivery. Advanced drug delivery reviews 65(9): 1148-1171.

8. Ramanathan S, Block LH (2001) The use of chitosan gels as matrices for electrically-modulated drug delivery. J Control Release 70(1-2): 109123.

ISSN: 2574-1241

DOI: 10.26717/BJSTR.2018.07.001512

Roche C de Guzman. Biomed J Sci \& Tech Res

(C) This work is licensed under Creative

Submission Link: https://biomedres.us/submit-manuscript.php
9. Murdan S (2003) Electro-responsive drug delivery from hydrogels. J Control Release 92(1-2): 1-17.

10. Tekin Celebi S, Solak AO, Ustundag Z, Demirci S (2012) Determination of $\mathrm{pK}$ a of benzoic acid- and $\mathrm{p}$-aminobenzoic acid-modified platinum surfaces by electrochemical and contact angle measurements. Chemical Papers 66(12):1146-1156.

11. Zarafonetis CJ, Dabich L, Negri D, Skovronski JJ, DeVol EB, et al. (1988) Retrospective studies in scleroderma: effect of potassium paraaminobenzoate on survival. J Clin Epidemiol 41(2): 193-205.

12. Kadhum WR, Oshizaka T, Ichiro H, Todo H, Sugibayashi K (2016) Usefulness of liquid-crystal oral formulations to enhance the bioavailability and skin tissue targeting of p-amino benzoic acid as a model compound. Eur J Pharm Sci 88: 282-290.

13. Ito S, Maruta K, Imai Y, Kato T, Ito M, et al. (1982) Urinary p-aminobenzoic acid determined in the pancreatic function test by liquid chromatography, with electrochemical detection. Clin Chem 28(2): 323-326.

14. Sheen B, De Guzman RC (2017) Electroresponsive PEG-chitosan matrix for anion release. Biomaterials and Tissue Technology 1(2): 1-6.

15. Ratner S, Blanchard M, Green DE (1946) Isolation of a peptide of paraaminobenzoic acid from yeast. J Biol Chem 164(2): 691-701.

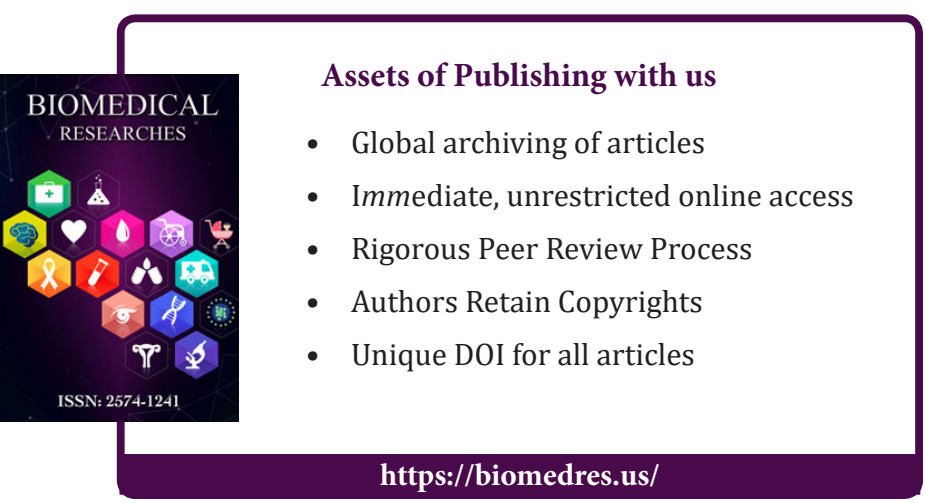

